\title{
Article \\ The Frequency Fluctuation Model for the van der Waals Broadening
}

\author{
Valery Astapenko ${ }^{1}$, Andrei Letunov ${ }^{* 2,3}$ and Valery Lisitsa ${ }^{1,2,3}$ \\ 1 Moscow Institute of Physics and Technology (National Research University), Institutskij Per. 9, \\ 141700 Dolgoprudnyj, Moscow Region, Russia; \\ 2 National Research Centre "Kurchatov Institute", 123182 Moscow, Russia; \\ 3 National Research Nuclear University MEPhI, 115409 Moscow, Russia; \\ * Correspondence:letunovandrey11@yandex.ru;
}

\begin{abstract}
The effect of atomic and molecular microfied dynamics on spectral line shapes is under consideration. This problem is treated in the framework of the Frequency Fluctuation Model (FFM). For the first time the FFM is tested for the broadening of a spectral line by neutral particles. The usage of the FFM allows one to derive simple analytical expressions and perform fast calculations of the intensity profile. The obtained results was compared with Chen and Takeo theory (CT), which is in a good agreement with experimental data. It was demonstrated that for moderate values of temperature and density the FFM successfully describes the effect of the microfield dynamics on a spectral line shape.
\end{abstract}

Keywords: Spectroscopy; Stark broadening; Atomic physics

\section{Introduction}

The problem of the microfield dynamics effect on a spectral line shape was recognized many years ago [1,2]. The best agreement with the results of Molecular Dynamics (MD) simulations provides the Frequency Fluctuation Model (FFM) [3]. It is based on dividing of a spectral line contour in a static field into separate regions, between which there is an exchange of intensities due to thermal motion. This model is widely used for spectral line shape calculations in plasmas (see e.g. [4-9]). It was shown that the FFM is equivalent to the method of the quantum kinetic equation [10]. This approach makes it possible to reformulate the FFM in terms of analytical expressions. Namely, the FFM spectral line shape can be considered as the functional of the static profile. This circumstance allows one to use simple analytical expressions and perform fast calculations of a spectral line shape for arbitrary values of temperatures and densities.

The FFM was formulated for spectral line shape calculations in plasmas. It was never used for the Stark broadening by neutral particles-to the best of our knowledge. Most of material on the van der Waals broadening accumulated by the end of 1960s is presented in the famous review of S. Chen and M. Takeo [11]. Moreover, in this paper the authors presented the line shape calculation method (based on the work of P.W. Anderson and J.D. Talman [12]) for arbitrary values of temperatures and densities. The Chen and Takeo results (CT) are very old. Nevertheless, it is in a good agreement with experimental data and can be used for the examination of the FFM theory. Moreover, the analytical expressions for the line shape presented in [11] are very cumbersome. The direct use of the CT theory in numerical calculations requires significant computational resources. So, the FFM may provide simple analytical expressions for the description of the van der Waals broadening.

The FFM has a drawback. It doesn't reproduce the impact width correctly, as it was directly shown in $[8,13]$. However, this problem was solved for the linear Stark effect. The resolution consists in the dependence of the jumping frequency, which characterizes 
the rate of change of the microfield, on the energy shift. This dependence was obtained by comparing of the analytical calculations with MD in the paper [8]. The authors of the work [13] overcame this problem in the alternative way. They used the asymptotic expression of the jumping frequency obtained by S. Chandrasekhar and J. von Neumann [14], who derived this for the description of the stellar dynamics.

The detailed analysis of the FFM results for different plasma parameters is presented in the paper [13]. It was demonstrated that the account of the dependence of the jumping frequency on the energy shift leads to the correct behaviour of the spectral line width in the impact limit for the linear Stark effect. The difference between the modified and the original versions of the FFM for moderate values of temperatures and densities is insignificant. So, account of the jumping frequency dependence on the field strength yields the simple analytical theory which provides the correct results for a wide range of plasma parameters. The situation is more complicated for the quadratic Stark effect. In this case the problem of reproducing the impact width wasn't solved in this way. However, for realistic plasma parameters the FFM with the constant jumping frequency provides quite satisfactory results.

Usually, the van der Waals broadening can be described in terms of the impact approximation [15]. So, the main goal of the present paper is the examination of the FFM for the van der Waals interaction. The second aim of this work is to provide the simple analytical algorithm for the line shape calculations for low temperatures (less that $300 \mathrm{~K}$ ) or high densities.

\section{Description of the method}

In the present paper we are focusing on the spectral broadening by neutral atoms and molecules. The detailed description of the analytical FFM formulation and the discussion of the the dynamics effect on the spectral line formation are given in [13]. For convenience, an abridged version is presented below.

The effect of a multiparticle electric field on molecular and atomic spectra is characterized by the ratio of the jumping frequency and the Stark shift

$$
v=\frac{\Omega_{J}}{\Omega_{S}^{n}},
$$

where

$$
\begin{gathered}
\Omega_{J}=N^{1 / 3} v_{T}, \\
\Omega_{S}^{n}=C_{n}^{S} N^{\frac{n}{3}} .
\end{gathered}
$$

Here, $N$ and $v_{T}$ are, respectively, the density and the thermal velocity of interacting particles; $C_{n}^{S}$ is the constant of the Stark effect; $\Omega_{J}$ is called the jumping frequency. The potential of binary interacting particles has the following form

$$
V(r)=\frac{C_{n}^{S}}{R^{n}} .
$$

In the present paper we consider the case of $n=6$, which corresponds to a wide class of van der Waals interactions.

The transition from binary to non-binary type of interactions is often characterized by the number of particles is the Weisskopf sphere $h$. For $n=6$ in the expression (4) it equals to [15]

$$
h=\left(\frac{3 \pi}{8} \frac{C_{6}^{S}}{v_{T}}\right)^{3 / 5} N .
$$

The parameter (5) also determines the transition from the static theory to the impact limit. The number of particles in the Weisskopf sphere is connected with $v$ by the simple relation

$$
h \sim v^{-3 / 5}
$$


Using the results from [15] we can estimate the parameter $v$. For $T \approx 300-5000 \mathrm{~K}$ $v$ approximately equals to

$$
v \approx\left(10^{35}-10^{38}\right) N^{-5 / 3}
$$

where $N$ is expressed in $\mathrm{cm}^{-3}$.

All over the paper atomic units are used $^{1}$. Also, for the sake of simplicity, we use the reduced detuning: $z=\Delta \omega / \Omega_{S}^{n}$.

The FFM procedure is equivalent to the method of the kinetic equation with strongcollision integral, which describes an intensity exchange between different regions of a static profile. [10]. The frequency of the exchange equals to $v$. The solution of this equation leads to the following expression for the resulting profile

$$
I(z)=\frac{1}{\pi} \operatorname{Re} \frac{\int \frac{W\left(z^{\prime}\right) d z^{\prime}}{v+i\left(z-z^{\prime}\right)}}{1-v \int \frac{W\left(z^{\prime}\right) d z^{\prime}}{v+i\left(z-z^{\prime}\right)}}
$$

where $W(z)$ is the normalized static profile. Note, that when $v \rightarrow 0 I(z)$ turns into $W(z)$.

The formula (8) can be rewritten in more convenient form

$$
I(z)=\frac{v}{\pi} \frac{\left.J_{0}(z) J_{2}(z)-J_{1}^{2}(z)\right)}{J_{2}^{2}(z)+v^{2} J_{1}^{2}(z)},
$$

where

$$
J_{k}(z)=\int_{-\infty}^{+\infty} \frac{W\left(z^{\prime}\right)\left(z-z^{\prime}\right)^{k} d z^{\prime}}{v^{2}+\left(z-z^{\prime}\right)^{2}}
$$

There is a useful relation between the functions $J_{0}(z)$ and $J_{2}(z)$

$$
J_{2}(z)=1-v^{2} J_{0}(z)
$$

S. Chen and M. Takeo presented the analytical expression for the intensity profile for an arbitrary value of $v$ for the van der Waals broadening [11] :

$$
I^{(C T)}(z)=C \cdot \operatorname{Re}\left\{\int_{-\infty}^{\infty} \exp \left[i z \tau-\frac{1}{v^{3 / 5}} \psi\left(v^{6 / 5} \tau\right)\right] d \tau\right\}
$$

where

$$
C=\left[\int_{-\infty}^{+\infty} \operatorname{Re}\left\{\int_{-\infty}^{\infty} \exp \left[i z \tau-\frac{1}{v^{3 / 5}} \psi\left(v^{6 / 5} \tau\right)\right] d \tau\right\} d z\right]^{-1}
$$

The function $\psi(x)$ can be approximated by the simple functions:

$$
\left\{\begin{array}{l}
\psi(x \ll 1)=\frac{(2 \pi)^{3 / 2}}{3} \sqrt{x}(1-i) \\
\psi(x \gg 1)=\frac{2 \pi}{5}\left(\frac{3 \pi}{8}\right)^{-3 / 5} \Gamma\left(\frac{2}{5}\right)\left(\cos \frac{\pi}{5}-i \sin \frac{\pi}{5}\right) x+1.91+2.63 i
\end{array}\right.
$$

where $\Gamma(z)$ is the gamma-function.

The expression (12) turns into the static profile $W(z)$ when $v$ approaches zero. In order to derive $W(z)$ it is necessary to use the approximation for $\psi(x)$ for small values of $x$.

\footnotetext{
$1 e=m=\hbar=1$, where $e$ - the elementary charge, $m$ - the mass of an electron, $\hbar$ is the Planck constant.
} 
Using the first relation of (13) and the stationary phase approximation one obtains the following formula for the static profile

$$
W(z)=\left\{\begin{array}{l}
0, z \geq 0 \\
\frac{2 \pi}{3|z|^{3 / 2}} \exp \left[\frac{4 \pi^{3}}{9 z}\right], z<0
\end{array}\right.
$$
[16].

The result (14) is in agreement with the well-known formula derived by H.Margenau

The examination of the FFM consists in the comparsion of the CT formula (12) with the expression (9). In the integrand of (10) we will substitute the static profile (14). In the impact limit $(v \gg 1)$ we can do a simple estimation of the impact width. Namely, for large values of $v$ the spectral line shape turns into the Lorentzian

$$
L(z)=\frac{1}{\pi} \frac{\gamma}{\gamma^{2}+\left(z-z_{0}\right)^{2}}
$$

where $\gamma$ is the width of the profile and $z_{0}$ is the coordinate of the center of the Lorentz profile.

Note, that the CT theory reproduces the impact limit. Indeed, the usage of the second relation from (13) leads to the expression (15).

In order to estimate the impact width for the FFM we will use the following property of a wide class of normalized profiles:

$$
F(0) \sim \frac{1}{\gamma}
$$

The relation (16) is valid here, because in the impact limit $\gamma$ and $z_{0}$ always have the same dependence on $v$ (see e.g. [15]). So, it is obvious that the property (16) works for the Lorentz profile (15). Using the asymptotic behavior of the formula (14) we can estimate $J_{k}(0)$ :

$$
\begin{gathered}
J_{0}(0) \sim \frac{1}{v^{2}} \\
J_{1}(0) \sim \int_{v}^{+\infty} d z^{\prime} \frac{1}{z^{\prime / 2}\left(z^{\prime 2}+v^{2}\right)} \sim \frac{1}{v^{3 / 2}}, \\
J_{2}(0) \sim \int_{v}^{+\infty} d z^{\prime} \frac{z^{1 / 2}}{z^{\prime 2}+v^{2}} \sim \frac{1}{v^{1 / 2}} \\
I(0) \sim \frac{1}{v^{1 / 2}} .
\end{gathered}
$$

From the relations (16) and (17) it is easy to see that $\gamma=\gamma_{F F M} \sim v^{1 / 2}$. Thus, the FFM doesn't reproduce the behavior of the profile width in the impact limit. Indeed, it is the well-know result of the impact theory [15] is $\gamma=8.16 v^{3 / 5}$. The difference in the dependence of the impact width on the power of velocity is very small $\gamma_{\text {impact }} / \gamma_{F F M} \sim$ $v^{1 / 10}$.

\section{The results of numerical calculations}

In order to test the FFM procedure we will compare it with the CT results. The numerical calculations shows that for $v \sim 1$ the FFM is in a good agreement with CT. The example of it is demonstrated in the figure (1), where one can see the comparsion of two theories for $v=1.5$. However, the FFM doesn't reproduce the impact width correctly (fig. 2) as it was shown in the previous section by the analytical estimations. The comparsion of the CT theory (in the impact limit) with the FFM profile is presented 


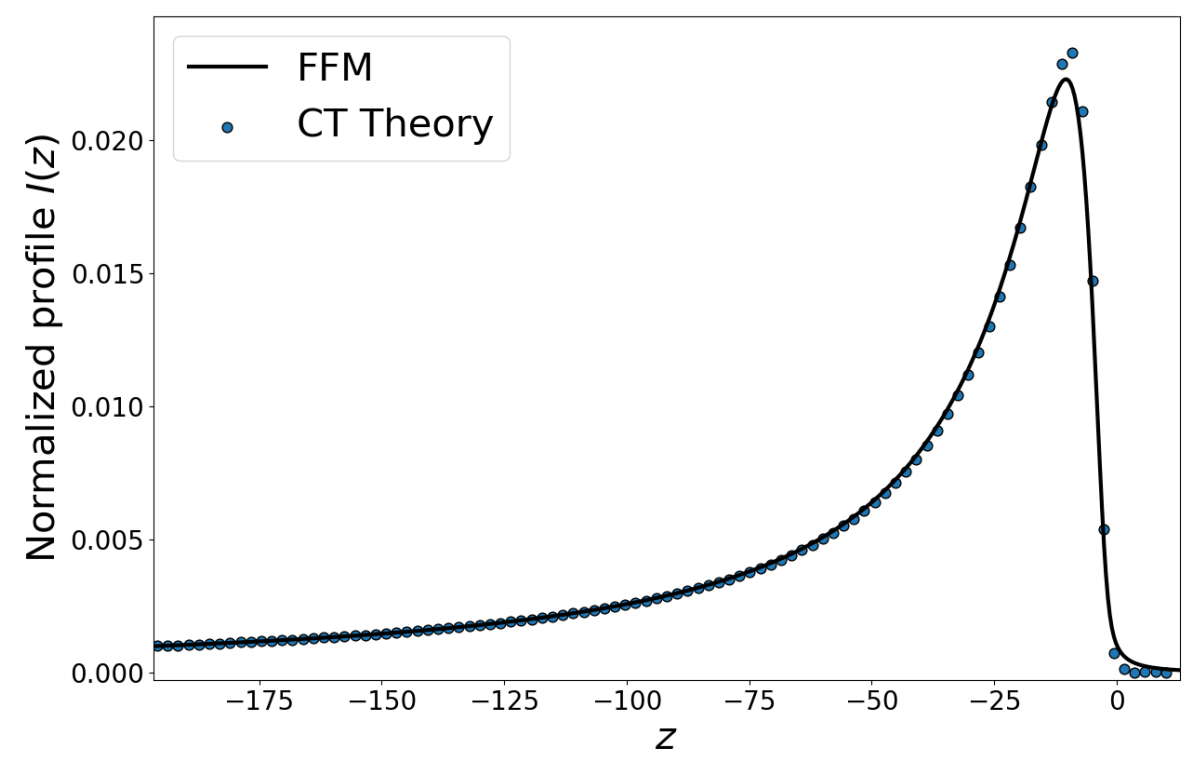

Figure 1. The normalized intensity profile of one spectral component as the function of the reduced energy shift. Comparison of the FFM profile with the Chen and Takeo theory; $v=1.5$

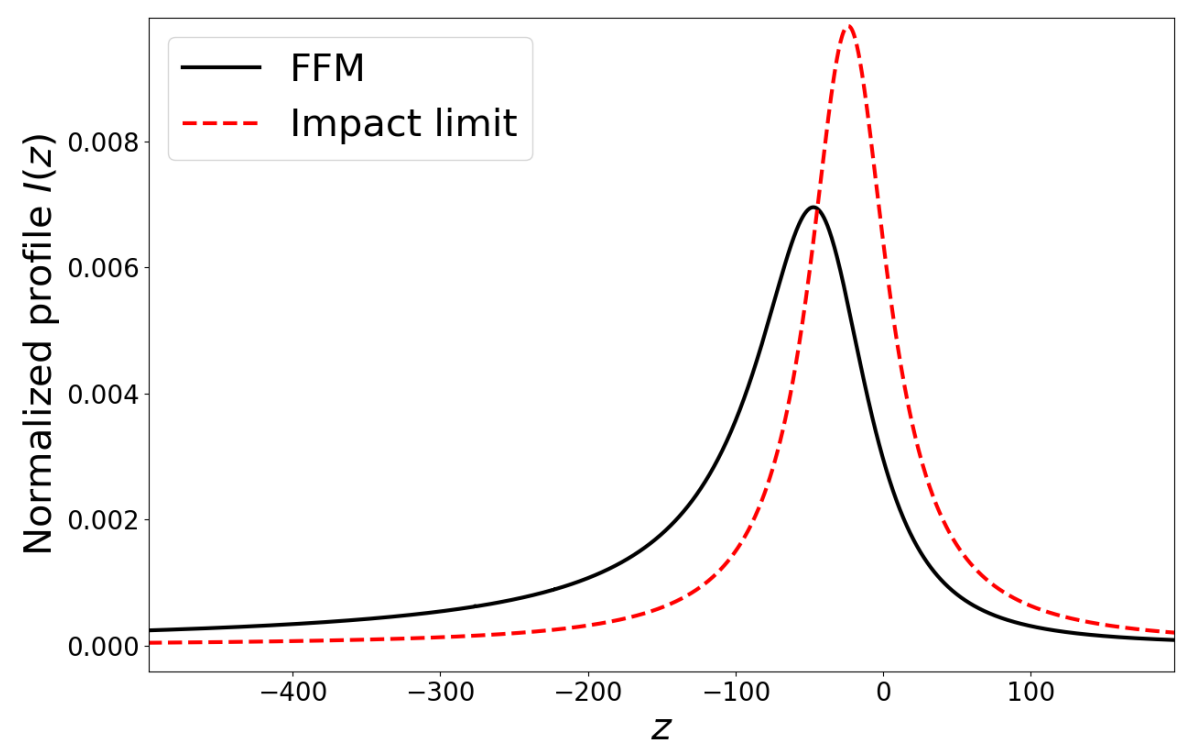

Figure 2. The normalized intensity profile of one spectral component as the function of the reduced energy shift. Comparison of the FFM profile with the impact theory; $v=100$

in the figure (2). The full width at half maximum of the FFM profile is approximately 1.5 as larger as the impact width for $v=100$. With an increase in the parameter $v$, the FFM profile slowly becomes wider than the impact line shape. This circumstance is connected with the slow growth of the ratio: $\gamma_{\text {impact }} / \gamma_{F F M} \sim v^{1 / 10}$. Moreover, we know only how $\gamma_{F F M}$ depend on $v$. Uncertainty in the value of the prefactor plays an important role in the impact width determination. However, derivation of the certain value of the FFM impact width prefactor by analytical calculations is a complex problem. It might be done numerically as in the work [13], but it isn't reasonable to do it until the dependence of the jumping frequency on the detuning will be determined. The discrepancy between 


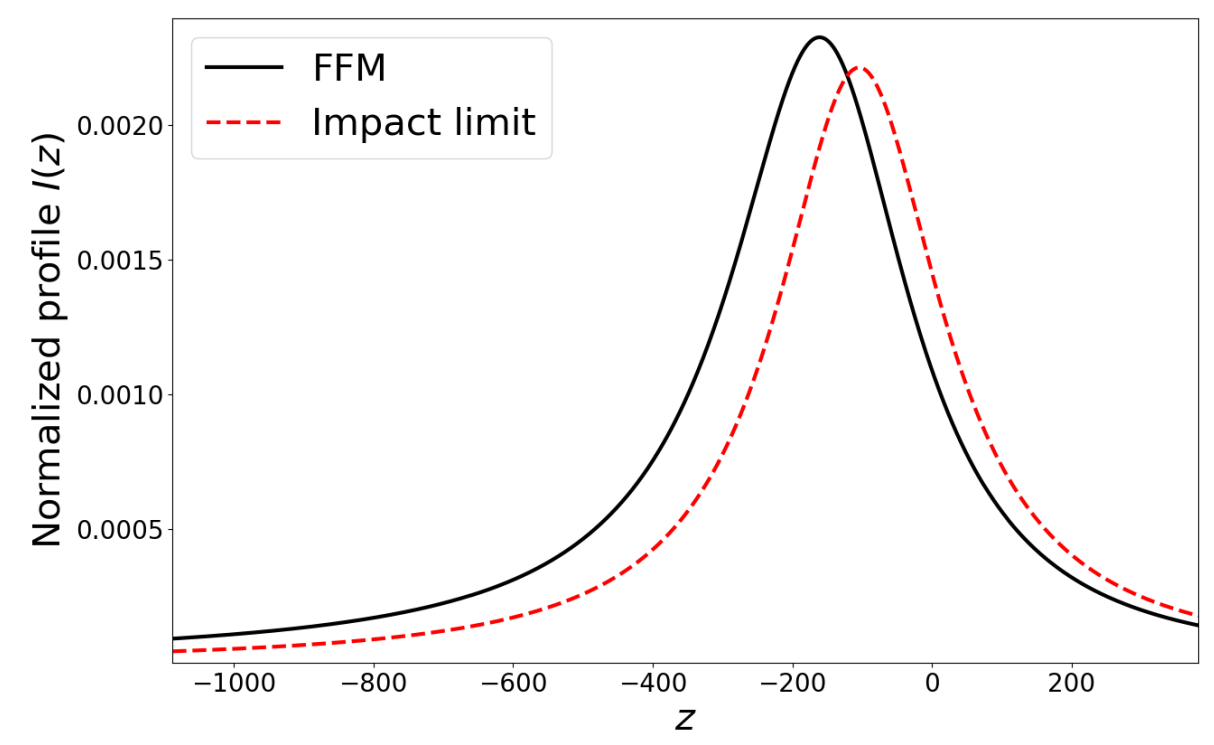

Figure 3. The normalized intensity profile of one spectral component as the function of the reduced energy shift. Comparison of the FFM profile with the impact theory; $v=1200$

two graphs becomes insignificant for $v \sim 1000$ (figure 3). According to the estimations (7) this value of $v$ for $T=300-5000 \mathrm{~K}$ corresponds to $N=10^{19}-10^{21} \mathrm{~cm}^{-3}$.

The discrepancy in the impact limit most likely might be eliminated by account of the dependence of the jumping frequency on the detuning. It was shown that for the linear Stark effect it leads to the correct result for large values of $v$ [13]. However, in order to determine this dependence it is necessary to describe complicated dynamics of the van der Waals forces.

\section{Conclusion}

The construction of the general analytical theory, which describes the effect of the complicated microfield dynamics on the spectral line shape formation, faces the great difficulties. The Frequency Fluctuation Model is believed to be the most accurate and simplest method for account such effects. So, it is necessary to examine this theory for different types of interractions. In the present paper we tested the FFM for the van der Waals forces.

The FFM shows good results for moderate values of temperature and density. The case of broadening by neutral atoms is no exception. The results of the FFM procedure are in agreement with CT theory (fig. 1).

The FFM doesn't reproduce the impact width correctly (fig. 2). As it was shown in [13], for the linear Stark effect this problem can be solved by account of the dependence of the jumping frequency on the field strength. Apparently, the resolution of the problem of the broadening by neutral particles consists in the description of the complicated dynamics of the van der Waals field.

Author Contributions: Conceptualization, V.L, V.A. and A.L.; methodology, V.L.; software, A.L.; validation, V.L., A.L. and V.A ; formal analysis, V.L.,V.A. and A.L.; investigation, A.L.,V.A. and V.L; resources, A.L.; data curation, V.L.; writing—original draft preparation, A.L.; writing-review and editing, V.L.; visualization, A.L.; supervision, V.L.; project administration, V.L.

Funding: This research received no external funding.

Institutional Review Board Statement: Not applicable

Informed Consent Statement: Not applicable 
Conflicts of Interest: The authors declare no conflict of interest.

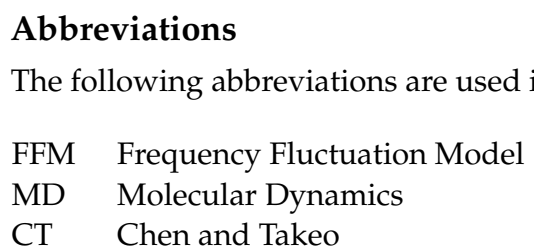

\section{References}

1. Griem, H.; Baranger, M.; Kolb, A.; Oertel, G. Stark broadening of neutral helium lines in a plasma. Physical Review 1962, $125,177$.

2. Kogan, V. Broadening of spectral lines in high-temperature plasma. Plasma physics and the problem of controlled fusion 1958.

3. Talin, B.; Calisti, A.; Godbert, L.; Stamm, R.; Lee, R.; Klein, L. Frequency-fluctuation model for line-shape calculations in plasma spectroscopy. Physical Review A 1995, 51, 1918.

4. Mossé, C.; Calisti, A.; Stamm, R.; Talin, B.; Bureyeva, L.; Lisitsa, V. A universal approach to Rydberg spectral line shapes in plasmas. Journal of Physics B: Atomic, Molecular and Optical Physics 2004, 37, 1343.

5. Calisti, A.; Bureyeva, L.; Lisitsa, V.; Shuvaev, D.; Talin, B. Coupling and ionization effects on hydrogen spectral line shapes in dense plasmas. The European Physical Journal D 2007, 42, 387-392.

6. Calisti, A.; Mossé, C.; Ferri, S.; Talin, B.; Rosmej, F.; Bureyeva, L.; Lisitsa, V. Dynamic Stark broadening as the Dicke narrowing effect. Physical Review E 2010, 81, 016406.

7. Ferri, S.; Calisti, A.; Mossé, C.; Mouret, L.; Talin, B.; Gigosos, M.A.; González, M.A.; Lisitsa, V. Frequency-fluctuation model applied to Stark-Zeeman spectral line shapes in plasmas. Physical Review E 2011, 84, 026407.

8. Stambulchik, E.; Maron, Y. Quasicontiguous frequency-fluctuation model for calculation of hydrogen and hydrogenlike Starkbroadened line shapes in plasmas. Physical Review E 2013, 87, 053108.

9. Letunov, A.; Lisitsa, V. The Coulomb Symmetry and a Universal Representation of Rydberg Spectral Line Shapes in Magnetized Plasmas. Symmetry 2020, 12, 1922.

10. Bureeva, L.; Kadomtsev, M.; Levashova, M.; Lisitsa, V.; Calisti, A.; Talin, B.; Rosmej, F. Equivalence of the method of the kinetic equation and the fluctuating-frequency method in the theory of the broadening of spectral lines. JETP letters 2010, 90, 647-650.

11. Takeo, M.; Chen, S. Broadening and shift of spectral lines due to the presence of foreign gases. Reviews of modern physics 1957, $29,20$.

12. Anderson, P.; Talman, J. Proceedings on the Conference on Broadening of Spectral Lines 1956.

13. Astapenko, V.; Letunov, A.; Lisitsa, V. From the Vector to Scalar Perturbations Addition in the Stark Broadening Theory of Spectral Lines. Universe 2021, 7, 176.

14. Chandrasekhar, S.; von Neumann, J. The Statistics of the Gravitational Field Arising from a Random Distribution of Stars. Astrophysical Journal 1942, 95, 489-531.

15. Sobelman, I.I. Introduction to the Theory of Atomic Spectra: International Series of Monographs in Natural Philosophy; Vol. 40, Elsevier, 2016.

16. Margenau, H. Theory of pressure effects of foreign gases on spectral lines. Physical Review 1935, $48,755$. 